\title{
Research on Attitude Representations and Attitude Differential Equations
}

\author{
Xiaoyu Wang and Xiaofeng $\mathrm{He}^{*}$ \\ College of Artificial Intelligence, National University of Defense Technology, Changsha 410000, China \\ ${ }^{*}$ Corresponding author
}

\begin{abstract}
Attitude estimation is used to determine the spatial orientation of an object relative to a reference coordinate frame, or to provide a method for vector conversion between the reference coordinate frame and the target coordinate frame. In this paper, after the definitions of 4 kinds of attitude representations, we derive the conversion formulas between different attitude representations. Then, a complete derivation process of the attitude differential equations is provided. In the end, we describe the result of a typical experiment, which demonstrates the correctness of these conversion formulas. All these researches can help understand the meaning of the attitude accurately and master the attitude updating algorithm in different fields of applications.
\end{abstract}

Keywords—attitude; conversion; quaternion; rotation

\section{INTRODUCTION}

Attitude estimation is used to determine the spatial orientation of an object relative to a reference coordinate frame, or to provide a method for vector conversion between the reference coordinate frame and the target coordinate frame. It has been applicated in many areas such as robot control, navigation, and computer vision. Attitude can be expressed in four different forms: (1) Euler angles, (2) direction cosine matrix, (3) attitude rotation vector, and (4) attitude quaternion[1]. Each of these forms is briefly described below. Without loss of generality, we choose the navigation frame (for simplicity, denoted as $\mathrm{n}$-frame) as the reference coordinate frame, and choose the body coordinate frame (denoted as bframe) as the target coordinate frame. The navigation frame is a local geographic frame which has its origin at the location of the navigation system, and axes aligned with the directions of north, east and the local vertical(down). The body frame is an orthogonal axis set which is aligned with the roll, pitch and yaw axes of the vehicle in which the navigation system is installed.

\section{A. Euler Angles}

The transformation from $n$-frame to $b$-frame can be carried out as 3 consecutive rotations about different axes: (1) rotate through angle $\psi$ about n-frame z-axis; (2) rotate through angle $\theta$ about new y-axis; (3) rotate through angle $\phi$ about new x-axis, where $\psi, \theta, \phi$ are called Euler rotation angles. Euler angles are written here in component form as follows: $[\phi, \theta, \psi]^{T}$.

\section{B. Direction Cosine Matrix}

The direction cosine matrix is a $3 \times 3$ matrix, the columns of which represent unit vectors in $b$-frame axes projected along the $n$-frame axes. Direction cosine matrix is written here in component form as follows:

$$
\boldsymbol{C}_{b}^{n}=\left[\begin{array}{lll}
c_{11} & c_{12} & c_{13} \\
c_{21} & c_{22} & c_{23} \\
c_{31} & c_{32} & c_{33}
\end{array}\right]
$$

A vector defined in b-frame, $\boldsymbol{r}^{b}$, may be expressed in $n$ frame by pre-multiplying the vector by the direction cosine matrix as follows:

$$
\boldsymbol{r}^{n}=\boldsymbol{C}_{b}^{n} \boldsymbol{r}^{b}
$$

\section{Rotation Vector}

The rotation vector, denoted here by the symbol $\boldsymbol{u}$, defines an axis of rotation and magnitude for rotation about the axis. Euler's theorem tells us that the transformation from n-frame to b-frame can be effected by a single rotation about an angle vector through an angle equal to the vector magnitude. This vector is called rotation vector.

\section{Attitude Quaternion}

The attitude quaternion, denoted here by the symbol $\boldsymbol{Q}$, is a 4-parameter representation of attitude. Quaternion can be expressed in different forms, we use the matrix form and the complex number form in this paper: $\boldsymbol{Q}=\left[q_{0}, q_{1}, q_{2}, q_{3}\right]^{T}=$ $q_{0}+\boldsymbol{i} q_{1}+\boldsymbol{j} q_{2}+\boldsymbol{k} q_{3}$. The attitude quaternion is corresponding to the rotation vector:

$$
\boldsymbol{Q}=\cos (u / 2)+(\boldsymbol{u} / u) \sin (u / 2)
$$

Where $u$ is the magnitude of rotation vector $\boldsymbol{u}$. A vector defined in b-frame, $\boldsymbol{r}^{b}$, may be expressed in n-frame as $\boldsymbol{r}^{n}$ using the quaternion directly. Treat $\boldsymbol{r}^{b}$ and $\boldsymbol{r}^{n}$ as zero-scalar quaternions, then we have:

$$
\boldsymbol{r}^{n}=\boldsymbol{Q} \otimes \boldsymbol{r}^{b} \otimes \boldsymbol{Q}^{*}
$$


Where $\boldsymbol{Q}^{*}$ is the complex conjugate of $\boldsymbol{Q}$, and the symbol $\otimes$ indicates quaternion multiplication[2].

\section{CONVERSION BETWEEN DifFERENT ATTITUDE FoRMS}

A. Euler Angles to Direction Cosine Matrix[3]

$$
\begin{aligned}
& \mathbf{C}_{b}^{n}= \\
& {\left[\begin{array}{cll}
\cos \theta \cos \psi & (-\cos \phi \sin \psi & (\sin \phi \sin \psi \\
& +\sin \phi \sin \theta \cos \psi) & +\cos \phi \sin \theta \cos \psi) \\
\cos \theta \sin \psi & (\cos \phi \cos \psi & (-\sin \phi \cos \psi \\
& +\sin \phi \sin \theta \sin \psi) & +\cos \phi \sin \theta \sin \psi) \\
-\sin \theta & \sin \phi \cos \theta & \cos \phi \cos \theta
\end{array}\right]}
\end{aligned}
$$

B. Euler Angles to Attitude Quaternion[3]

$$
\boldsymbol{Q}=\left[\begin{array}{l}
\cos \left(\frac{\phi}{2}\right) \cos \left(\frac{\theta}{2}\right) \cos \left(\frac{\psi}{2}\right)+\sin \left(\frac{\phi}{2}\right) \sin \left(\frac{\theta}{2}\right) \sin \left(\frac{\psi}{2}\right) \\
\sin \left(\frac{\phi}{2}\right) \cos \left(\frac{\theta}{2}\right) \cos \left(\frac{\psi}{2}\right)-\cos \left(\frac{\phi}{2}\right) \sin \left(\frac{\theta}{2}\right) \sin \left(\frac{\psi}{2}\right) \\
\cos \left(\frac{\phi}{2}\right) \sin \left(\frac{\theta}{2}\right) \cos \left(\frac{\psi}{2}\right)+\sin \left(\frac{\phi}{2}\right) \cos \left(\frac{\theta}{2}\right) \sin \left(\frac{\psi}{2}\right) \\
\cos \left(\frac{\phi}{2}\right) \cos \left(\frac{\theta}{2}\right) \sin \left(\frac{\psi}{2}\right)-\sin \left(\frac{\phi}{2}\right) \sin \left(\frac{\theta}{2}\right) \cos \left(\frac{\psi}{2}\right)
\end{array}\right]
$$

C. Direction Cosine Matrix to Euler Angles

$$
\begin{aligned}
& \psi=\left\{\begin{array}{c}
\arctan \left[c_{21} / c_{11}\right],\left(c_{11}>0\right) \\
\operatorname{sign}\left(c_{21}\right) \frac{\pi}{2},\left(c_{11}=0\right) \\
\arctan \left[c_{21} / c_{11}\right]-\pi,\left(c_{11}<0, c_{21}<0\right) \\
\arctan \left[c_{21} / c_{11}\right]+\pi,\left(c_{11}<0, c_{21} \geq 0\right)
\end{array}\right. \\
& \theta=\arcsin \left(-c_{31}\right) \\
& \phi=\arctan \left[c_{32} / c_{33}\right]
\end{aligned}
$$

\section{Direction Cosine Matrix to Rotation Vector}

Rotation vector $\boldsymbol{u}$ can rotate $\mathrm{n}$-frame to b-frame. Because the rotation is always around $\boldsymbol{u}$, it's easy to know that the coordinates of $\boldsymbol{u}$ in n-frame and b-frame are the same. That is to say: $\boldsymbol{u}^{n}=\boldsymbol{u}^{b}$ and $\boldsymbol{u}^{n}=\boldsymbol{C}_{b}^{n} \boldsymbol{u}^{b}=\boldsymbol{C}_{b}^{n} \boldsymbol{u}^{n}$. Hence, the vector $\boldsymbol{u}^{n}$ is one of the eigenvectors of $\boldsymbol{C}_{b}^{n}$, corresponding to the eigenvalue 1. When $\boldsymbol{C}_{b}^{n} \neq I_{3}$, the dimension of the eigenspace of eigenvalue 1 is one[4]. Suppose we have obtained a eigenvector $\boldsymbol{v}$ which is corresponding to eigenvalue 1 , then

$$
\boldsymbol{u} / u=v /|\boldsymbol{v}|
$$

$$
u=2 \arccos \left(\sqrt{1+c_{11}+c_{22}+c_{33}} / 2\right)
$$

Hence

$$
\boldsymbol{u}=2 \arccos \left(\sqrt{1+c_{11}+c_{22}+c_{33}} / 2\right)(\boldsymbol{v} /|\boldsymbol{v}|)
$$

\section{E. Direction Cosine Matrix to Attitude Quaternion[3]}

For small angular displacements, the attitude quaternion may be derived using the following relationships:

$$
\boldsymbol{Q}=\left[\begin{array}{l}
q_{0} \\
q_{1} \\
q_{2} \\
q_{3}
\end{array}\right]=\left[\begin{array}{c}
\sqrt{\left(1+c_{11}+c_{22}+c_{33}\right)} / 2 \\
\left(c_{32}-c_{23}\right) /\left(4 q_{0}\right) \\
\left(c_{13}-c_{31}\right) /\left(4 q_{0}\right) \\
\left(c_{21}-c_{12}\right) /\left(4 q_{0}\right)
\end{array}\right]
$$

A more comprehensive algorithm for the extraction of attitude quaternion parameters from the direction cosine matrix is described by Shepperd[5].

\section{F. Rotation Vector to Direction Cosine Matrix[2]}

$$
\left.\boldsymbol{C}_{b}^{n}=\boldsymbol{I}_{3}+(\sin u / u)\lfloor\boldsymbol{u} \times\rfloor+\left((1-\cos u) / u^{2}\right)\lfloor\boldsymbol{u} \times\rfloor \boldsymbol{u} \times\right\rfloor
$$

\section{G. Rotation Vector to Attitude Quaternion}

$$
\boldsymbol{Q}=\cos (u / 2)+\sin (u / 2) \boldsymbol{u} / u
$$

H. Attitude Quaternion to Direction Cosine Matrix[2]

$$
\boldsymbol{C}_{b}^{n}=\left[\begin{array}{lll}
1-2\left(q_{2}^{2}+q_{3}^{2}\right) & 2\left(q_{1} q_{2}-q_{0} q_{3}\right) & 2\left(q_{1} q_{3}+q_{0} q_{2}\right) \\
2\left(q_{1} q_{2}+q_{0} q_{3}\right) & 1-2\left(q_{1}^{2}+q_{3}^{2}\right) & 2\left(q_{2} q_{3}-q_{0} q_{1}\right) \\
2\left(q_{1} q_{3}-q_{0} q_{2}\right) & 2\left(q_{2} q_{3}+q_{0} q_{1}\right) & 1-2\left(q_{1}^{2}+q_{2}^{2}\right)
\end{array}\right]
$$

\section{Attitude Quaternion to Rotation Vector}

It can be known from (13) that the vector $\boldsymbol{u}$ is parallel to the vector $\left[q_{1}, q_{2}, q_{3}\right]^{T}$, and $q_{0}=\cos (u / 2)$, hence

$$
\boldsymbol{u} / u=\left[q_{1}, q_{2}, q_{3}\right]^{T} / \sqrt{q_{1}^{2}+q_{2}^{2}+q_{3}^{2}}
$$

$$
\boldsymbol{u}=\left(2 \arccos \left(q_{0}\right) / \sqrt{q_{1}^{2}+q_{2}^{2}+q_{3}^{2}}\right)\left[q_{1}, q_{2}, q_{3}\right]^{T}
$$

Where 


\section{J. Brief Summary}

In this section, we provide the conversions between different attitude representations, they are:

$$
\begin{aligned}
& {[\phi, \theta, \psi]^{T} \rightarrow \boldsymbol{Q}} \\
& \text { b \ b } \\
& C_{b}^{n} \leftrightarrow u
\end{aligned}
$$

\section{Derivation of AtTitude Differential EQUATIONS}

\section{A. Euler Angles Differential Equation}

The following relationship exists between Euler angles $[\phi, \theta$, $\psi]^{T}$ and relative angular rates $\boldsymbol{\omega}_{n b}^{b}$ :

$$
\left[\begin{array}{c}
\omega_{n b, x}^{b} \\
\omega_{n b, \mathrm{y}}^{b} \\
\omega_{n b, \mathrm{z}}^{b}
\end{array}\right]=\left[\begin{array}{c}
\phi \\
0 \\
0
\end{array}\right]+\boldsymbol{C}_{\phi}\left[\begin{array}{l}
0 \\
\theta \\
0
\end{array}\right]+\boldsymbol{C}_{\phi} \boldsymbol{C}_{\theta}\left[\begin{array}{l}
0 \\
0 \\
\psi
\end{array}\right]
$$

This equation can be rearranged and expressed as follows:

$$
\left[\begin{array}{l}
\phi \\
\theta \\
\psi
\end{array}\right]=\frac{1}{\cos \theta}\left[\begin{array}{ccc}
\cos \theta & \sin \phi \sin \theta & \cos \phi \sin \theta \\
0 & \cos \phi \cos \theta & -\sin \phi \cos \theta \\
0 & \sin \phi & \cos \phi
\end{array}\right]\left[\begin{array}{c}
\omega_{n b, x}^{b} \\
\omega_{n b, \mathrm{y}}^{b} \\
\omega_{n b, \mathrm{~b}}^{b}
\end{array}\right]
$$

(18) may be solved in a strapdown system to update the Euler rotations of the body with respect to the navigation frame However, their use is limited since the solution of the roll angle and yaw angle become indeterminate when $\theta= \pm(\pi / 2)[3]$.

\section{B. Direction Cosine Matrix Differential Equation}

Suppose $\boldsymbol{R}_{b}$ is a vector that is attached to b-frame, so

$$
\left.\frac{\mathrm{d} \boldsymbol{R}_{b}}{\mathrm{~d} t}\right|_{b}=\boldsymbol{R}_{b}^{b}=\boldsymbol{0}_{3 \times 1}
$$

Hence

$$
\left.\frac{\mathrm{d} \boldsymbol{R}_{b}}{\mathrm{~d} t}\right|_{n}=\left.\frac{\mathrm{d} \boldsymbol{R}_{b}}{\mathrm{~d} t}\right|_{b}+\omega_{n b} \times \boldsymbol{R}_{b}=\boldsymbol{\omega}_{n b} \times \boldsymbol{R}_{b}
$$

Project (20) into n-frame, we have

$$
\boldsymbol{R}_{b}^{n}=\left\lfloor\boldsymbol{\omega}_{n b}^{n} \times\right\rfloor \boldsymbol{R}_{b}^{n}=\left\lfloor\boldsymbol{\omega}_{n b}^{n} \times\right\rfloor \boldsymbol{C}_{b}^{n} \boldsymbol{R}_{b}^{b}
$$

Differentiate $\boldsymbol{R}_{b}^{n}=\boldsymbol{C}_{b}^{n} \boldsymbol{R}_{b}^{b}$ at both ends

$$
\boldsymbol{R}_{b}^{n}=\boldsymbol{C}_{b}^{n} \boldsymbol{R}_{b}^{b}+\boldsymbol{C}_{b}^{n} \boldsymbol{R}_{b}^{b}=\boldsymbol{C}_{b}^{n} \boldsymbol{R}_{b}^{b}
$$

Comparing (21) with (22), we have

$$
\left\lfloor\omega_{n b}^{n} \times\right\rfloor \boldsymbol{C}_{b}^{n} \boldsymbol{R}_{b}^{b}=\boldsymbol{C}_{b}^{n} \boldsymbol{R}_{b}^{b}
$$

Because $\boldsymbol{R}_{b}^{b}$ can be arbitrarily selected, we have

$$
\boldsymbol{C}_{b}^{n}=\left\lfloor\boldsymbol{\omega}_{n b}^{n} \times\right\rfloor \boldsymbol{C}_{b}^{n}=\boldsymbol{C}_{b}^{n}\left\lfloor\boldsymbol{\omega}_{n b}^{b} \times\right\rfloor \boldsymbol{C}_{n}^{b} \boldsymbol{C}_{b}^{n}=\boldsymbol{C}_{b}^{n}\left\lfloor\boldsymbol{\omega}_{n b}^{b} \times\right\rfloor
$$

\section{Attitude Quaternion Differential Equation}

Suppose $\boldsymbol{R}_{b}$ is a vector that is attached to b-frame. According to (21), we have

$$
\boldsymbol{R}_{b}^{n}=\left\lfloor\omega_{n b}^{n} \times\right\rfloor \boldsymbol{R}_{b}^{n}
$$

Additionally, we have

$$
\boldsymbol{R}_{b}^{n}=\left[\begin{array}{c}
0,1,0,0 \\
0,0,1,0 \\
0,0,0,1
\end{array}\right]\left\{\boldsymbol{Q} \otimes\left[\begin{array}{c}
0 \\
\boldsymbol{R}_{b}^{b}
\end{array}\right] \otimes \boldsymbol{Q}^{*}\right\}
$$

Differentiate (26) at both ends, and replacing $\boldsymbol{R}_{b}^{b}$ with $\boldsymbol{0}_{3 \times 1}$, we have

$$
\boldsymbol{R}_{b}^{n}=\left[\begin{array}{c}
0,1,0,0 \\
0,0,1,0 \\
0,0,0,1
\end{array}\right]\left\{\boldsymbol{Q} \otimes\left[\begin{array}{c}
0 \\
\boldsymbol{R}_{b}^{b}
\end{array}\right] \otimes \boldsymbol{Q}^{*}+\boldsymbol{Q} \otimes\left[\begin{array}{c}
0 \\
\boldsymbol{R}_{b}^{b}
\end{array}\right] \otimes \boldsymbol{Q}^{*}\right\}
$$

Since

$$
\boldsymbol{Q} * \otimes \boldsymbol{Q}=\left[\begin{array}{llll}
1 & 0 & 0 & 0
\end{array}\right]^{T}
$$

and

$$
\left[\begin{array}{c}
0 \\
\boldsymbol{R}_{b}^{n}
\end{array}\right]=\boldsymbol{Q} \otimes\left[\begin{array}{c}
0 \\
\boldsymbol{R}_{b}^{b}
\end{array}\right] \otimes \boldsymbol{Q}^{*}
$$

We have

$$
\begin{aligned}
& \boldsymbol{R}_{b}^{n}=\left[\begin{array}{c}
0,1,0,0 \\
0,0,1,0 \\
0,0,0,1
\end{array}\right]\left[\boldsymbol{Q} \otimes \boldsymbol{Q}^{*} \otimes\left(\boldsymbol{Q} \otimes\left[\begin{array}{c}
0 \\
\boldsymbol{R}_{b}^{b}
\end{array}\right] \otimes \boldsymbol{Q}^{*}\right)\right]+ \\
& {\left[\begin{array}{c}
0,1,0,0 \\
0,0,1,0 \\
0,0,0,1
\end{array}\right]\left[\left(\boldsymbol{Q} \otimes\left[\begin{array}{c}
0 \\
\boldsymbol{R}_{b}^{b}
\end{array}\right] \otimes \boldsymbol{Q}^{*}\right) \otimes \boldsymbol{Q} \otimes \boldsymbol{Q}^{*}\right]} \\
& =\left[\begin{array}{c}
0,1,0,0 \\
0,0,1,0 \\
0,0,0,1
\end{array}\right]\left\{\boldsymbol{Q} \otimes \boldsymbol{Q}^{*} \otimes\left[\begin{array}{c}
0 \\
\boldsymbol{R}_{b}^{n}
\end{array}\right]+\left[\begin{array}{c}
0 \\
\boldsymbol{R}_{b}^{n}
\end{array}\right] \otimes \boldsymbol{Q} \otimes \boldsymbol{Q}^{*}\right\}
\end{aligned}
$$


Differentiate (28) at both ends

$$
\boldsymbol{Q} \otimes \boldsymbol{Q}^{*}+\boldsymbol{Q} \otimes \boldsymbol{Q}^{*}=\left[\begin{array}{llll}
0 & 0 & 0 & 0
\end{array}\right]^{T}
$$

Hence

$$
\boldsymbol{Q} \otimes \boldsymbol{Q}^{*}=-\boldsymbol{Q} \otimes \boldsymbol{Q}^{*}
$$

Taking (32) into (30), we get

$$
\boldsymbol{R}_{b}^{n}=\left[\begin{array}{c}
0,1,0,0 \\
0,0,1,0 \\
0,0,0,1
\end{array}\right]\left\{\boldsymbol{Q} \otimes \boldsymbol{Q}^{* \otimes}\left[\begin{array}{c}
0 \\
\boldsymbol{R}_{b}^{n}
\end{array}\right]-\left[\begin{array}{c}
0 \\
\boldsymbol{R}_{b}^{n}
\end{array}\right] \otimes \boldsymbol{Q} \otimes \boldsymbol{Q}^{*}\right\}
$$

Suppose $\boldsymbol{Q}=\left[q_{0}, q_{1}, q_{2}, q_{3}\right]^{T}$, then the complex conjugate of $\boldsymbol{Q}$ is $\boldsymbol{Q}^{*}=\left[q_{0},-q_{1},-q_{2},-q_{3}\right]^{T}$, and

$$
q_{0}^{2}+q_{1}^{2}+q_{2}^{2}+q_{3}^{2}=1
$$

Differentiate (34) at both ends, we can obtain

$$
q_{0} q_{0}+q_{1} q_{1}+q_{2} q_{2}+q_{3} q_{3}=0
$$

Hence

$$
\boldsymbol{Q} \otimes \boldsymbol{Q}^{*}=\left[\begin{array}{c}
0 \\
q_{0} q_{1}-q_{1} q_{0}+q_{2} q_{3}-q_{3} q_{2} \\
q_{0} q_{2}-q_{1} q_{3}-q_{2} q_{0}+q_{3} q_{1} \\
q_{0} q_{3}+q_{1} q_{2}-q_{2} q_{1}-q_{3} q_{0}
\end{array}\right]
$$

Let

$$
\boldsymbol{Q} \otimes \boldsymbol{Q}^{*}=\left[0, v_{1}, v_{2}, v_{3}\right]^{T}=\left[0, \boldsymbol{v}^{T}\right]^{T}
$$

So

$$
\left[\begin{array}{c}
0 \\
\boldsymbol{R}_{b}^{n}
\end{array}\right] \otimes \boldsymbol{Q} \otimes \boldsymbol{Q}^{*}=\left[\begin{array}{c}
0 \\
\boldsymbol{R}_{b}^{n}
\end{array}\right] \otimes\left[\begin{array}{l}
0 \\
\boldsymbol{v}
\end{array}\right]=\left[\begin{array}{c}
-\boldsymbol{R}_{b}^{n} \bullet \boldsymbol{v} \\
\boldsymbol{R}_{b}^{n} \times \boldsymbol{v}
\end{array}\right]
$$

$$
\boldsymbol{Q} \otimes \boldsymbol{Q} * \otimes\left[\begin{array}{c}
0 \\
\boldsymbol{R}_{b}^{n}
\end{array}\right]=\left[\begin{array}{c}
0 \\
\boldsymbol{v}
\end{array}\right] \otimes\left[\begin{array}{c}
0 \\
\boldsymbol{R}_{b}^{n}
\end{array}\right]=\left[\begin{array}{c}
-\boldsymbol{v} \bullet \boldsymbol{R}_{b}^{n} \\
\boldsymbol{v} \times \boldsymbol{R}_{b}^{n}
\end{array}\right]=\left[\begin{array}{c}
-\boldsymbol{R}_{b}^{n} \bullet \boldsymbol{v} \\
-\boldsymbol{R}_{b}^{n} \times \boldsymbol{v}
\end{array}\right](39)
$$

Taking (38) and (39) into (33), we have

$$
\boldsymbol{R}_{b}^{n}=\left[\begin{array}{c}
0,1,0,0 \\
0,0,1,0 \\
0,0,0,1
\end{array}\right]\left\{\left[\begin{array}{l}
-\boldsymbol{R}_{b}^{n} \bullet \boldsymbol{v} \\
-\boldsymbol{R}_{b}^{n} \times \boldsymbol{v}
\end{array}\right]-\left[\begin{array}{c}
-\boldsymbol{R}_{b}^{n} \bullet \boldsymbol{v} \\
\boldsymbol{R}_{b}^{n} \times \boldsymbol{v}
\end{array}\right]\right\}=\boldsymbol{R}_{b}^{n} \times(-2 \boldsymbol{v})(40)
$$

According to (25) and (40), we have

$$
\boldsymbol{R}_{b}^{n} \times(-2 \boldsymbol{v})=\left\lfloor\boldsymbol{\omega}_{n b}^{n} \times\right\rfloor \boldsymbol{R}_{b}^{n}=\boldsymbol{R}_{b}^{n} \times\left(-\boldsymbol{\omega}_{n b}^{n}\right)
$$

Because $\boldsymbol{R}_{b}^{b}$ can be arbitrarily selected, (41) is established for any $\boldsymbol{R}_{b}^{n}$, we have

$$
2 v=\omega_{n b}^{n}
$$

Hence

$$
\begin{aligned}
& \boldsymbol{Q} \otimes \boldsymbol{Q}^{*}=\left[\begin{array}{l}
0 \\
\boldsymbol{v}
\end{array}\right]=\frac{1}{2}\left[\begin{array}{c}
0 \\
\boldsymbol{\omega}_{n b}^{n}
\end{array}\right] \Rightarrow \boldsymbol{Q}=\frac{1}{2}\left[\begin{array}{c}
0 \\
\boldsymbol{\omega}_{n b}^{n}
\end{array}\right] \otimes \boldsymbol{Q} \\
& \Rightarrow \boldsymbol{Q}=\frac{1}{2} \boldsymbol{Q} \otimes\left[\begin{array}{c}
0 \\
\boldsymbol{\omega}_{n b}^{b}
\end{array}\right] \otimes \boldsymbol{Q} * \otimes \boldsymbol{Q}=\frac{1}{2} \boldsymbol{Q} \otimes\left[\begin{array}{c}
0 \\
\boldsymbol{\omega}_{n b}^{b}
\end{array}\right]
\end{aligned}
$$

Treat $\omega_{n b}^{b}$ as a zero-scalar quaternion, then we have

$$
\boldsymbol{Q}=\frac{1}{2} \boldsymbol{Q} \otimes \boldsymbol{\omega}_{n b}^{b}
$$

D. Rotation Vector Differential Equation

Let $f_{1}=\cos (u / 2), f_{2}=\sin (u / 2) / u$, so

$$
\boldsymbol{Q}=f_{1}+f_{2} \boldsymbol{u}
$$

Taking (45) into (44)

$$
\begin{aligned}
& \boldsymbol{Q}=\left(\left(f_{1}+f_{2} \boldsymbol{u}\right) / 2\right) \otimes \boldsymbol{\omega}_{n b}^{b}=f_{1} \boldsymbol{\omega}_{n b}^{b} / 2+f_{2} \boldsymbol{u} \otimes \boldsymbol{\omega}_{n b}^{b} / 2 \\
& =f_{1} \boldsymbol{\omega}_{n b}^{b} / 2+f_{2}\left(-\boldsymbol{u} \bullet \boldsymbol{\omega}_{n b}^{b}+\boldsymbol{u} \times \boldsymbol{\omega}_{n b}^{b}\right) / 2
\end{aligned}
$$

Differentiate equation (45) at both ends

$$
\boldsymbol{Q}=f_{1}+f_{2} \boldsymbol{u}+f_{2} \boldsymbol{u}=-u u f_{2} / 2+u\left(f_{1} / 2-f_{2}\right) \boldsymbol{u} / u+f_{2} \boldsymbol{u}(47)
$$

According (46) and (47), we have

$$
\boldsymbol{u}=\frac{1}{2} u u-\frac{1}{2} \boldsymbol{u} \bullet \boldsymbol{\omega}_{n b}^{b}-\frac{u}{u}\left(\frac{1}{2} \frac{f_{1}}{f_{2}}-1\right) \boldsymbol{u}+\frac{1}{2} \frac{f_{1}}{f_{2}} \boldsymbol{\omega}_{n b}^{b}+\frac{1}{2} \boldsymbol{u} \times \boldsymbol{\omega}_{n b}^{b}
$$

Since $\boldsymbol{u}$ is a zero-scalar quaternion, so we have 


$$
\frac{1}{2} u u-\frac{1}{2} \boldsymbol{u} \bullet \boldsymbol{\omega}_{n b}^{b}=0 \Rightarrow \frac{u}{u}=\frac{1}{u^{2}} \boldsymbol{u} \bullet \boldsymbol{\omega}_{n b}^{b}
$$

Hence

$$
\begin{aligned}
& \boldsymbol{u}=-\frac{u}{u}\left(\frac{1}{2} \frac{f_{1}}{f_{2}}-1\right) \boldsymbol{u}+\frac{1}{2} \frac{f_{1}}{f_{2}} \boldsymbol{\omega}_{n b}^{b}+\frac{1}{2} \boldsymbol{u} \times \boldsymbol{\omega}_{n b}^{b} \\
& =-\frac{1}{u^{2}}\left(\frac{1}{2} \frac{f_{1}}{f_{2}}-1\right)\left(\boldsymbol{u} \bullet \boldsymbol{\omega}_{n b}^{b}\right) \boldsymbol{u}+\frac{1}{2} \frac{f_{1}}{f_{2}} \boldsymbol{\omega}_{n b}^{b}+\frac{1}{2} \boldsymbol{u} \times \boldsymbol{\omega}_{n b}^{b}
\end{aligned}
$$

According to the identities of vector, we have

$$
\left(\boldsymbol{u} \bullet \boldsymbol{\omega}_{n b}^{b}\right) \boldsymbol{u}=\boldsymbol{u} \times\left(\boldsymbol{u} \times \boldsymbol{\omega}_{n b}^{b}\right)+u^{2} \boldsymbol{\omega}_{n b}^{b}
$$

Taking (51) into (50), we have

$$
\begin{aligned}
& \boldsymbol{u}=\boldsymbol{\omega}_{n b}^{b}+\frac{1}{2} \boldsymbol{u} \times \boldsymbol{\omega}_{n b}^{b}+\frac{1}{u^{2}}\left(1-\frac{1}{2} \frac{f_{1}}{f_{2}}\right) \boldsymbol{u} \times\left(\boldsymbol{u} \times \boldsymbol{\omega}_{n b}^{b}\right) \\
& =\boldsymbol{\omega}_{n b}^{b}+\frac{1}{2} \boldsymbol{u} \times \boldsymbol{\omega}_{n b}^{b}+\frac{1}{u^{2}}\left(1-\frac{u \sin (u)}{2(1-\cos (u))}\right) \boldsymbol{u} \times\left(\boldsymbol{u} \times \boldsymbol{\omega}_{n b}^{b}\right)
\end{aligned}
$$

\section{E. Brief Summary}

In this section, we provide the complete derivation process of the attitude differential equations, especially the Attitude Quaternion Differential Equation and Rotation Vector Differential Equation. These differential equation may be used to update attitude. On how to solve these differential equations, readers can refer to [2] and [7].

\section{EXPERIMENTS}

In order to verify the correctness of the mutual conversion formulas between different attitude representation forms, we use MATLAB to conduct experiments. We generate some attitude Euler angle vectors randomly, and use these Euler angle vectors to calculate attitude representations of other form. Then the errors that exist between the representations (obtained through different computing processes) of the same attitude form can be calculated. For simplicity, we use additive error instead of multiplicative error. The experimental results show that the errors is less than $10^{-14}$, which proves the correctness of these conversion formulas. Due to the limited space, we give only one typical experiment result in TABLE I. In this table, the Eul(Euler angles) was generated randomly, and was used to calculate the DCM(direction cosine matrix), then the DCM was

\begin{tabular}{|c|c|}
\hline Attitude & Results \\
\hline Eul & {$[-1.4586,1.0968,2.7269]^{T}$} \\
\hline DCM & {$\left[\begin{array}{ccc}-0.4177, & 0.7641, & -0.4916 \\
0.1839, & -0.4587, & -0.8693 \\
-0.8898, & -0.4536, & 0.0511\end{array}\right]$} \\
\hline RVT & {$[1.3840,1.3254,-1.9312]^{T}$} \\
\hline Quat & {$[0.2089,0.4975,0.4764,-0.6942]^{T}$} \\
\hline RVT_1 & {$[1.3840,1.3254,-1.9312]^{T}$} \\
\hline DCM_1 & {$\left[\begin{array}{ccc}-0.4177, & 0.7641, & -0.4916 \\
0.1839, & -0.4587, & -0.8693 \\
-0.8898, & -0.4536, & 0.0511\end{array}\right]$} \\
\hline Eul_1 & {$[-1.4586,1.0968,2.7269]^{T}$} \\
\hline Quat_1 & {$[0.2089,0.4975,0.4764,-0.6942]^{T}$} \\
\hline Eul-Eul_1 & $(1.0 \mathrm{e}-14) *[0.1332,-0.0444,0.1332]^{T}$ \\
\hline DCM - DCM_1 & $(1.0 \mathrm{e}-15) *\left[\begin{array}{ccc}-0.6661 & -0.4441 & 0.1665 \\
-0.3608 & 0 & 0.2220 \\
0.2220 & -0.3331 & 0.6870\end{array}\right]$ \\
\hline RVT - RVT_1 & {$[0,0,0]^{T}$} \\
\hline Quat-Quat_1 & $(1.0 \mathrm{e}-16) *[0.8327,0,0,0]^{T}$ \\
\hline
\end{tabular}
used to calculate RVT(rotation vector). Other representations was calculated similarly. We use additive error instead of multiplicative error to show the correctness of the mutual conversion formulas.
TABLE I. EXPERIMENT RESULT

\section{CONCLUDING REMARKS}

In this paper, we first give the definitions of 4 kinds of attitude representations, and then derive the conversion formulas between the different attitude representations. Finally, we provide the complete derivation process of the attitude differential equations. All these researches can help understand the meaning of the attitude accurately and master the attitude updating algorithm in different fields of application.

\section{REFERENCES}

[1] Xiaoping Hu. Navigation technology foundation[M]. National Defense Industry Press, 2015.

[2] Yongyuan Qin. Inertial Navigation[M]. Science Press, 2014.

[3] Titterton D H, Weston J L. Strapdown inertial navigation technology[M]. Institution of Electrical Engineers, 2004.

[4] Zhangming He,Guiliang Feng. Eigenvalue Method for Solving Attitude Quaternion[J]. Journal of Changsha Univercity, 2010, 24(2):4-6.

[5] van DRIEL,N.:'A review of the status and capabilities of navstar-GPS', NLR TP 92042-U. 1992.

[6] Barfoot T, Forbes J R, Furgale P T. Pose estimation using linearized rotations and quaternion algebra[J]. Acta Astronautica, 2011, 68(12):101-112.

[7] Xiaoping Hu. Autonomous Navigation Technology[M]. National Defense Industry Press, 2016. 